\title{
Die Reformen der Hochschule als hegemoniales Projekt?
}

\author{
Studiengebühren und Bologna im geltenden Verfassungsrecht
}

»Entlassen wir Schulen und Hochschulen in die Freiheit «, ${ }^{\mathrm{I}}$ so die zentrale Forderung der hochschulpolitischen Reformdebatte der letzten Jahre. Es ist nicht zu übersehen, dass sich dahinter die zunehmende Ausrichtung der Hochschulorganisation und der Bildungsinhalte an Marktgesichtspunkten verbirgt: Studiengebühren, Bachelor/Master-Studium (»Bologna-Prozess«) und das Modell der Stiftungsuniversität bilden die Eckpfeiler dieser neuen Marktverfassung der Hochschulorganisation. Alle vornehmlich wissenschafts- und sozialpolitischen Einwände vermochten bislang nicht, die Reorganisation des Hochschulwesens nachhaltig zu beeinflussen. Das liegt nicht zuletzt daran, dass dieser Prozess als Element der aktuell emergierenden hegemonialen Konstellation, ob sie nun als Postmoderne, Postfordismus oder Neoliberalismus bezeichnet wird, zu begreifen ist. ${ }^{2}$

Gleichzeitig verweisen aktuelle Gerichtsurteile und eine zunehmende Beschäftigung der juristischen Literatur darauf, dass die hegemoniale Auseinandersetzung um die Zukunft des Hochschulwesens auch im Rechtssystem ausgetragen wird. Hier können die wissenschafts- und sozialpolitischen Erwägungen, die in der politischen Debatte gegen einen Umbau der Hochschulen eingebracht werden, an geltendes Recht in Form von gesellschaftlichen Grundrechten anknüpfen. Diese Bruchstellen ermöglichen gegenhegemonialen Positionen, sich in den Kampf um das Recht und mithin um gesellschaftliche Hegemonie einzubringen. ${ }^{3}$ Die vor diesem Hintergrund auftretenden rechtlichen Auseinandersetzungen, betreffen vor allem die verfassungsrechtlichen Möglichkeiten und Grenzen der Einführung von Studiengebühren (hierzu I.) sowie der Umsetzung der Bologna-Kriterien (hierzu 2.).

\section{Rechtskollisionen: Studiengebühren vs. (globale) Grundrechte}

Eine wesentliche Bedeutung bei der rechtlichen Auseinandersetzung um Studiengebühren kommt der Frage zu, ob die entsprechenden Gesetze mit Art. I3 Abs. 2 c) UN-Sozialpakt (IPwskR) kollidieren, was deren Verfassungswidrigkeit zur Folge hätte. ${ }^{4}$ Gemäß Art. I 3 Abs. 2 c) IPwskR erkennen die Vertragsstaaten an, dass im Hinblick auf die volle Verwirklichung des dort niedergelegten Rechts auf Bildung der Hochschulunterricht auf jede geeignete Weise,

I Roman Herzog, Entlassen wir Schulen und Hochschulen in die Freiheit, Frankfurter Rundschau vom 6.II.I997, Nr. 86, I4.

2 Zur Bedeutung der Universitäten als Hegemonieapparate in einem historischen Block vgl. Antonio Gramsci, Gefängnishefte. Kritische Gesamtausgabe, hrsg. v. Klaus Bochmann/Wolfgang Haug, Hamburg/Berlin I 991, Heft 6, $\mathbb{\int} 87,782$.

3 Zum Konzept einer rechtlichen Hegemonietheorie vgl. Sonja Buckel/Andreas Fischer-Lescano, Hegemonie im globalen Recht - Zur Aktualität der Gramscianischen Rechtstheorie, in: Hegemonie gepanzert mit Zwang - Zivilgesellschaft und Politik im Staatsverständnis Antonio Gramscis, Baden-Baden 2007, 8 5-104.

4 Dies resultiert aus Art. 3 I GG (vgl. Sven Söllner, Studiengebühren und das Recht auf Bildung, Mannheim 2007, I 2 I f. m.w.N.), bei kompetenzrechtlichen Bedenken hiergegen jedenfalls aus dem Grundsatz der Bundestreue gemäß Art. 28 Abs. 2 GG (vgl. Thorsten Deppner/Daniel Heck, Studiengebühren vor dem Hintergrund der Umsetzung völkerrechtlicher Verpflichtungen im Bundesstaat und der Vorgaben des materiellen Verfassungsrechts, NVwZ 2008, 45-48, 47 f. m.w.N.). 
»insbesondere durch allmähliche Einführung der Unentgeltlichkeit«, allgemein verfügbar und jedermann zugänglich gemacht werden muss.'

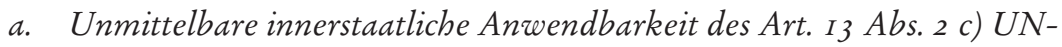 Sozialpakt}

Der Frage nach einer inhaltlichen Kollision der Studiengebührengesetze vorgelagert ist das Problem, ob diese völkerrechtliche Norm innerstaatlich anwendbar ist. ${ }^{6}$ Die unmittelbare Anwendbarkeit einer völkerrechtlichen Norm setzt voraus, dass sie sich nach Inhalt, Zweck und Wortlaut zur Rechtsanwendung eignet.7 Im Hinblick auf Art. I3 Abs. 2 c) IPwskR wird vom OVG Münster eingewandt, dass es der Norm an der hierfür erforderlichen Bestimmtheit fehle, da dem zweiten Halbsatz (»insbesondere«) nicht eindeutig zu entnehmen sei, ob es sich beim Gebührenverbot um eine zwingende Voraussetzung zur Verwirklichung von Hochschulzugangsgerechtigkeit handele oder lediglich um eine mögliche - wenn auch zentrale - Maßnahme hierfür. Dies werde durch diesbezüglich abweichende Auslegungsergebnisse in der juristischen Literatur belegt, so dass sich keine mit hinreichender rechtsstaatlicher Bestimmtheit aus dem Vertrag ableitbaren Kriterien über die völkerrechtliche Zulässigkeit der Einführung von Studiengebühren ableiten ließen. ${ }^{8}$

Diese Rechtsansicht findet im Hinblick auf die aktuelle Rechtsprechung und den juristischen Meinungsstand jedoch kaum Bezugspunkte im geltenden Recht. ${ }^{9}$ Dagegen wird insistiert, dass bereits an den vorliegend gewählten Prüfungsmaßstab der Bestimmtheit keine hohen Anforderungen zu stellen seien, da dieser als Element des Rechtsstaatsgrundsatzes (Art. 20 Abs. 3 GG) die BürgerInnen vor unvorhersehbaren Eingriffen der Staatsgewalt schützen solle, Art. I 3 Abs. 2 c) IPwskR mit dem Verbot der Einführung von Studiengebühren jedoch eine begünstigende Regelung beinhalte. ${ }^{10}$ Ferner entspreche die Vorschrift (»insbesondere «) der - auch im Hinblick auf andere Normen des UN-Sozialpakts - verbreiteten gesetzlichen Regelungstechnik, eine zwingende, lediglich nicht abschließende, Tatbestandsvoraussetzung zu nennen. ${ }^{\text {I }}$ Ebenfalls lasse das Vorliegen zahlreicher Auslegungsergebnisse nicht auf eine rechtsstaatlich bedenkliche Unbestimmtheit schließen, vielmehr zähle die Rechtsauslegung zu den anerkannten Tätigkeiten der Rechtsanwendung. ${ }^{\text {I2 }}$

\footnotetext{
5 Zur Vereinbarkeit der Studiengebührengesetze mit Art. I 2 I, 3 I GG, Art. 20 I (Sozialstaatsprinzip) vgl. VG Minden, DVBl. 2007, 773-780; VG Hannover, Urteil vom 8.6.2007 - 6 B 8296/06; VG Freiburg, VBlBW 2007, 426-438; VG Karlsruhe, Urteil vom I I.7.2007 - 7 K 444/07; OVG Münster, DVBl. 2007, I 442-I 448; kritisch dazu Deppner/Heck (Fn. 4), 48. Zur Vereinbarkeit der Studiengebührengesetze Hessens mit Art. 59 Abs. I HessVerf vgl. Urteil des Staatsgerichtshofes des Landes Hessen vom i r.6.2006, abrufbar (2.7.2008) über http://www.staatsgerichtshof.hessen.de. Vgl. dagegen Arndt Schmehl, Studiengebühren in Hessen verfassungsgemäß? - Die Verbürgung der Unentgeltlichkeit des Hochschulunterrichts und ihre Beschränkungen nach Art. 59 HessVerf, NVwZ 2006, 883-888.

6 Ein gem. Art. 59 Abs. 2 GG erforderliches Vertragsgesetz liegt vor, vgl. BGBl. I 973 II, I 569.

7 Vgl. BVerfGE 29, 348, 360.

8 OVG Münster (Fn. 5), I I 43-I I45; ähnlich Volker Haug, Rechtliche Möglichkeiten und Grenzen einer Einführung von Studienbeiträgen, WissR 33 (2000), I-2 I, 6.

9 Zur unmittelbaren Anwendbarkeit vgl. die erstinstanzlichen VG-Urteile (Fn. 5); ferner VG Frankfurt am Main, NJW-RR I999, 325, 330; BVerwG, Buchholz 421.2. Hochschulrecht Nr. $160=$ BeckRS 2004, 2 I 306; BVerfGE I I 2, 226, 245; zur Rechtsprechungsentwicklung vgl. Söllner (Fn. 4), I 45-I 47; Bodo Pieroth/Bernd Hartmann, Studienbeitragsdarlehen am Maßstab höherrangigen Rechts, NWVBl. 2007, 8I86, 8 I f.; Eibe Riedel/Sven Söllner, Studiengebühren im Lichte des UN-Sozialpakts, JZ 2006, 270-277, 272 f.; Söllner (Fn. 4), I 52-I6I; Manfred Zulegg, Der Internationale Pakt über wirtschaftliche, soziale und kulturelle Rechte, RdA I974, 32 I-332, 324.

Io Deppner/Heck (Fn. 4), 47.

I I Riedel/Söllner (Fn. 9), 272 f.

I 2 Vgl. BVerfGE 34, 269.
} 
Zentral für eine reformorientierte Rechtsauffassung bleibt die Ausarbeitung einer hegemonialen Begründung für eine Völkerrechtsmäßigkeit der Studiengebührengesetze. Dabei favorisieren die Verwaltungsgerichte und zahlreiche Literaturstimmen eine teleologische Reduktion des Art. I3 Abs. 2 c) IPwskR.'13 Indem die Regelung das Ziel der Verwirklichung von Hochschulzugangsgerechtigkeit verfolge und eine Unentgeltlichkeit des Studiums nur als beispielhaftes Instrument (»insbesondere«) dafür begreife, sei eine nicht am Wortlaut, sondern am eigentlichen Zweck der Vorschrift orientierte Sichtweise geboten. ${ }^{14}$ Mithin sei Art. I3 Abs. 2 c) IPwskR einschränkend dahin auszulegen, dass die Erhebung eines Entgelts für den Hochschulunterricht zulässig ist, wenn jeder nach seinen Fähigkeiten unabhängig von seiner sozialen Herkunft und seinen finanziellen Möglichkeiten einen chancengleichen Zugang zur Hochschulbildung hat. ${ }^{\text {Is }}$

Der Behauptung, dass bereits ein darlehenbasiertes Studiengebührenmodell einen chancengleichen Zugang zu Bildung gewährleiste, ist entgegenzusetzen, dass eine verwaltungsgerichtliche Auseinandersetzung mit der empirisch vielfach belegten Abschreckungswirkung von Studiengebühren auf einkommensschwache Studienanwärter bisher nicht erfolgt ist. ${ }^{16}$ Auf dieses Problem verweisen auch Rechtsauffassungen, die Art. I3 Abs. 2 c) IPwskR als Gleichheitsrecht begreifen, infolgedessen eine Einführung von Studiengebühren mit ihrer uneinheitlichen Wirkung auf Studierwillige - je nach deren Einkommenssituation - unbedingt völkerrechtswidrig sei. ${ }^{17}$

Weiterhin sieht sich die teleologische Reduktion auch verfassungsrechtlichen Bedenken ausgesetzt, denn allen verwaltungsgerichtlichen Urteilen fehlt der Nachweis, dass die Regelung planwidrig zu weit geraten ist, m.a.W. das regressive Verbot der Studiengebühren dem telos Hochschulzugangsgerechtigkeit zuwiderläuft. Eine dahingehende Argumentation findet sich lediglich bei Riedel/ Söllner: »In Deutschland hat die Abschaffung von Hochschulgebühren zu keiner höheren Bildungsbeteiligung geführt (...). Die soziale Bildungsgerechtigkeit ist in Deutschland schwächer ausgeprägt als in vergleichbaren Ländern mit Studiengebühren. Dies ist ein abschreckendes Beispiel dafür, dass die Einführung der Unentgeltlichkeit auch nach Jahrzehnten nicht zwingend zu dem gewünschten Ziel des sozial gerechten Hochschulzugangs führt. «1 ${ }^{18}$ Dass ein insgesamt sozial selektives Bildungssystem trotz einer Studiengebührenfreiheit im internationalen Vergleich schlecht abschneidet, liegt jedoch ebenso auf der Hand wie die Tatsache, dass dies vielmehr gegen eine Vereinbarkeit von Studiengebühren mit Art. I 3 Abs. 2 c) IPwskR gerade in Deutschland spricht. ${ }^{19}$

Das verweist auf den letzten Punkt, der den vielleicht Ausschlaggebenden für die vorgenannten Urteile bildet. Denn die teleologische Reduktion wird durch systematische Erwägungen im Hinblick auf ausbleibende Rügen des UN-Ausschusses bei der Einführung von Gebührenmodellen in anderen Ländern ergänzt. ${ }^{20}$ Dem wird entgegnet, dass in Bezug auf andere Staaten und vor allem

\footnotetext{
I 3 Vgl. Fn. 5; grundlegend dazu Riedel/Söllner (Fn. 9), 273 f.

I4 Vgl. ausdrücklich VG Karlsruhe (Fn. 5), Rn. 43.

Is So i.E. alle erstinstanzlichen VG-Urteile (Fn. s).

I6 Deppner/Heck (Fn. 4), 47.

I7 Stefan Lorenzmaier, Völkerrechtswidrigkeit der Einführung von Studiengebühren, NVwZ 2006, 759762,760 .

I 8 Riedel/Söllner (Fn. 9), 273.

I9 Ähnlich Deppner/Heck (Fn. 4), 47.

20 VG Minden (Fn. 5), 775; Pieroth/Hartmann (Fn. 9), 82; Riedel/Söllner (Fn. 9), 273.
} 
auf Deutschland das Gegenteil der Fall sei - den Grund hierfür haben Riedel/ Söllner mit ihrem Verweis auf die soziale Selektivität des deutschen Bildungssystems selbst genannt. ${ }^{21}$ Auch die teleologische Reduktion des Art. I3 Abs. 2 c) IPwskR bleibt ein äußerst fragwürdiges Argument.

\section{c. Fazit: (Aus-)Bildung als Wettbewerbsfaktor im juridischen Diskurs}

Die juridische Kritik an der Rechtsauffassung, die gesellschaftliche Grundrechte in Form des Art. I3 Abs. 2 c) IPwskR für unanwendbar erklärt, wenn sie der Reorganisation der Hochschule Grenzen setzen, manifestiert die rechtlichen Grenzen für reformorientierte Bestrebungen, insoweit sie sich zumindest im Rechtssystem nicht einfach entgegenstehenden - in Normen kondensierten Wertungen entledigen können. Gleichsam reflektiert bspw. diese Rechtsauffassung des OVG Münster ganz deutlich den politischen Zeitgeist, sozial- und wissenschaftspolitische Erwägungen Marktimperativen unterzuordnen. Dass auch die Verwaltungsgerichte Studiengebühren mit ihrer Wirkung, das Studium infolge dieses Investments als re-invest- bzw. vermarktungsausgerichtet zu begreifen, als notwendiges Element im globalen (Bildungs-)Wettbewerb auffassen, wird spätestens an der umfassenden Begründung der teleologischen Reduktion deutlich, die auf die völkerrechtliche Zulässigkeit von Studiengebühren in anderen Ländern verweist. Dennoch haben die aufgezeigten dogmatischen Inkonsistenzen gezeigt, dass diese Form des Umgangs mit dem völkerrechtlichen Normenbestand äußerst fragwürdig bleibt ${ }^{22}$ und dass der Kampf um die globalen sozialen Rechte erst am Anfang steht. ${ }^{23}$

\section{Der Bologna-Prozess und die JuristInnenausbildung}

Ein weiteres Element der emergierenden Marktverfassung der Hochschulorganisation bildet die Neuausrichtung der Hochschulbildung an den Bologna-Kriterien. $^{24}$ Ihr gleichsam rechtsunverbindlicher wie sachzwanghafter Charakter zeigt ihre Bedeutung innerhalb des aktuell hegemonialen Projekts. ${ }^{25}$

\section{a. „Wird der Juristenausbildung der Bologna-Prozess gemacht? «26}

Die dadurch ausgelöste Debatte hat sich auch in den stetigen Reformprozess der JuristInnenausbildung eingeschrieben. Zahlreiche Modelle einer Integration der Bologna-Kriterien in das bestehende Modell der JuristInnenausbildung liegen bereits vor. ${ }^{27} \mathrm{Nachstehend} \mathrm{kann} \mathrm{es} \mathrm{nicht} \mathrm{darum} \mathrm{gehen,} \mathrm{die} \mathrm{einzelnen} \mathrm{Modelle} \mathrm{zu}$ sezieren, um daraus einen eigenständigen Reformvorschlag zu entwickeln. Vielmehr soll sich auf einige Aspekte beschränkt werden, die - zumindest in einer kritischen Perspektive - bereits vorhandene Schwächen der JuristInnenausbildung weiter verschärfen.

2i Lorenzmaier (Fn. I7), 760 f.; Söllner (Fn. 4), S. I 77 f.

22 Dies wäre Prüfungsgegenstand einer Verfassungsbeschwerde, vgl. Lorenzmaier (Fn. 17), 762.

23 Generell siehe den Text von Thomas Seibert (in diesem Heft); ferner Jan Philip Wimalasena, Die Durchsetzung sozialer Menschenrechte. Rechtsfortbildung am Beispiel des Internationalen Sozialpakts von I966, KJ 2008, 2-23.

24 Vgl. http://www.bmbf.de/de/3336.php (Stand 2.7.2008).

25 Vgl. Matthias v. Wulffen, Der Bologna-Prozess und seine möglichen Auswirkungen auf die Justiz, NVwZ 2005, 890-895, 891.

26 Thomas Pfeiffer, Wird der Juristenausbildung der Bologna-Prozess gemacht?, NJW 2005, 228 I-2283.

27 Vgl. instruktiv Heribert Hirte/Sebastian Mock, Die Juristenausbildung in Europa vor dem Hintergrund des Bologna-Prozesses, JuS-Beilage 2005, 3-I4. 
Im Wesentlichen bezieht sich eine solche auf die Studieninhalte, genauer: auf den Schwerpunkt Fallbearbeitung und einer damit verbundenen strukturellen Überlastung mit Rechtsdogmatik zu Lasten der kritischen Reflexion. ${ }^{28}$ Zudem werden die gängigen Alltagspraktiken der JuristInnenausbildung (Disziplinierungsmechanismen in Form eines vergleichsweise niedrigen Notendurchschnitts, einer prinzipiellen Überforderung etc.) mit ihrer »Tendenz zu struktureller Verunsicherung sowie Förderung elitären Verhaltens $\ll^{29}$ thematisiert. Vor diesem Hintergrund sind die Reformvorschläge aus Bologna alles andere als eine Entwarnung: Das Bachelor-Studium mit seiner berufs- und arbeitsmarktorientierten Konzeption dürfte weitere Einschnitte bzgl. der grundlagen- und reflexionsorientierten Inhalte der JuristInnenausbildung begründen. Die bereits erfolgten Umsetzungen an den Universitäten Mannheim und Dresden zeigen die ausschließliche Verknüpfung von juristischen Bachelorstudiengängen mit betriebswirtschaftlichen Studieninhalten (»Law and Finance«) - andere gesellschaftliche Bezüge von Recht bleiben außen vor. Insoweit das anschließende Masterstudium nur den $30 \%$ Besten ermöglicht werden soll, $3^{\circ}$ verschärft diese zunehmende Selektion den tendenziell verunsichernden und elitebildenden Charakter der JuristInnenausbildung. ${ }^{3}$

\section{b. Rechtskollisionen: Bologna und die Wissenschaftsfreibeit}

Die Integration des Bolognaprozesses ist im Hinblick auf die damit verbundene ausbildungsorientierte Studienkonzeption und die wirtschaftsorientierten Studieninhalte als strukturelle Korruption der wissenschaftlichen Lehre durch Wirtschaft und Ökonomisierung zu begreifen. ${ }^{32}$

Einer insoweit nahe liegenden Rechtskollision zwischen den Bologna-Maßnahmen des nationalen Rechts und der Wissenschaftsfreiheit (Art. 5 Abs. 3 GG) mangelt es aufgrund der geltenden Grundrechtsdogmatik in Bezug auf die Hinnahme staatlichen Eingreifens in den Hochschulbetrieb an praktischer Relevanz: »Wie weit diese Hinnahme geht, ist mir bei einer Passage aufgefallen, die ich in einem sehr verbreiteten Lehrbuch der Grundrechte gelesen habe. Dort schreiben die Professoren Pieroth und Schlink zur Wissenschaftsfreiheit: >Die Rechtsprechung ist hierzu unergiebig; der abgehobene Charakter der Wissenschaft hat kein nennenswertes Konfliktpotential zwischen Wissenschaft und Gesellschaft und daher auch keine nennenswerten Eingriffsnotwendigkeiten aufkommen lassen.< Meine Damen und Herren, wenn selbst die über die Grundrechte schreibenden Universitätslehrer nicht bemerken (oder nichts dagegen haben), dass in ihrem eigenen Betrieb der Fremde schon zum Teilhaber geworden ist und deshalb gar nicht mehr >eingreifen muss, ist das System gegen Art. 5 immun. «33

Evtl. kann der Bologna-Prozess als Teil der heute bildungspolitisch hegemonialen strukturellen Korruption der Wissenschaft durch Wirtschaft und Ökonomisierung einer kritischen Rechtswissenschaft als Anknüpfungspunkt dienen, das

28 Ernst-Wolfgang Böckenförde, Juristenausbildung - auf dem Weg ins Abseits, JZ I997, 317-326.

29 Sonja Buckel, Die Mechanik der Macht in der Juristenausbildung, Kritische Justiz 2002, I I I-I I 4, I I 2.

30 Jens Jepp, Der Bologna-Prozess als Chance, NJW 2005, 2283-2286, 2283.

3 I In diesem Kontext ist auf den Widerstand gegen eine Aufgabe der Ersten Juristischen Staatsprüfung im Zuge des Bologna-Prozesses hinzuweisen. Vgl. Beate Merk, Der Bologna-Prozess - die Erste Juristische Staatsprüfung auf dem Prüfstand?, ZRP 2004, 264-266.

32 In Bezug auf die Forschung vgl. Gunther Teubner, Globale Zivilverfassungen - Alternativen zu einer staatszentrierten Verfassungstheorie, ZaöRV 2003, I-28, I7.

33 Axel Flessner, Deutsche Juristenausbildung - Die kleine Reform und die europäische Perspektive, JZ I 996, 689-696, $694 \mathrm{f}$. 

schaftsfreiheit zu sensibilisieren.

\title{
3. Fazit: Kritische Rechtswissenschaft als gegenhegemoniale Rechtspraxis
}

Die Ausführungen haben gezeigt, dass wissenschafts- und sozialpolitische Inhalte des geltenden Rechts Anknüpfungspunkte für gegenhegemoniale Strategien im Rechtssystem bieten. Durch diese positive Bezugnahme auf den Herrschaftsmodus Recht unterscheiden sich aktuelle Konzepte einer kritischen Rechtswissenschaft von früheren Analysen des Rechts, die vor allem dessen Verschleierungsfunktion in Bezug auf asymmetrische Machtverhältnisse thematisierten. ${ }^{34}$ Einen weiteren Unterschied bildet die transnationale Perspektive, die neben einer kritischen Begleitung im Hinblick auf demokratische Defizite rechtlicher Internationalisierung auch die Möglichkeit bietet, 35 auf transnationaler Ebene vorhandene Rechte einzuklagen - darauf verweist das vorliegend besprochene Beispiel der rechtlichen Auseinandersetzung um die Studiengebühren. ${ }^{6}$ Auch wenn eine rechtstheoretische Kritik an den undemokratischen und verschleiernden Prozessen der Rechtsfindung - das Beispiel der gerichtlichen Verfahren bzgl. der Studiengebühren verweist darauf - erforderlich bleibt, 37 ist es notwendig, gegenhegemoniale Positionen in die Rechtspraxis einzuschreiben. ${ }^{3}$

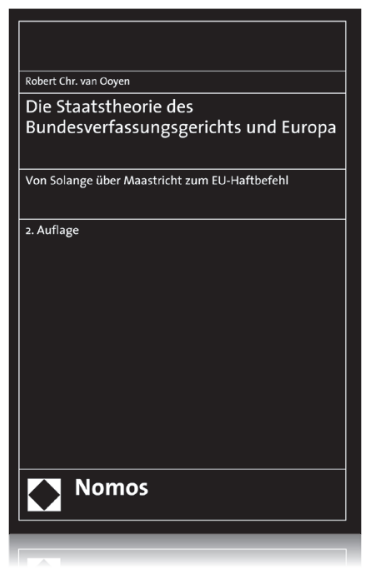

\section{Die Staatstheorie des Bundes- verfassungsgerichts und Europa}

\author{
Von Solange über Maastricht zum \\ EU-Haftbefehl
}

Von ORR Dr. Robert Chr. van Ooyen

2. Auflage 2008, 114 S., brosch., 19,- $€$, ISBN 978-3-8329-3556-6

Bitte bestellen Sie bei Ihrer Buchhandlung oder bei Nomos | Telefon 07221/2104-37| Fax-43 | www.nomos.de|sabine.horn@nomos.de

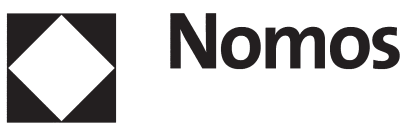

34 Vgl. instruktiv Sonja Buckel, Neo-Materialistische Rechtstheorie, in: dies. u.a. (Hrsg.), Neue Theorien des Rechts, Stuttgart 2006, I I 7-I 38 .

35 Vgl. Hauke Brunkhorst, Die Legitimationskrise der Weltgesellschaft, in: Matthias Albert u.a. (Hrsg.), Weltstaat und Weltstaatlichkeit - Beobachtungen globaler politischer Strukturbildung, Wiesbaden 2007, $63-107$.

36 Vgl. generell dazu Harold Hangju Koh, Transnational Public Law Litigation, in roo Yale L.J. (I99I), 2347-2402.

37 Vgl. dazu exemplarisch die Critical Legal Studies Bewegung, instruktiv Günter Frankenberg, Partisanen der Rechtskritik: Critical Legal Studies etc., in: Buckel (Fn. 34), 97-I I6.

38 Zum Konzept des organischen Intellektuellen vgl. Antonio Gramsci (Fn. 2), Heft I 2, \ I, I 500. 